University of Nebraska - Lincoln

DigitalCommons@University of Nebraska - Lincoln

Uniformed Services University of the Health

Sciences

U.S. Department of Defense

2011

Schistosoma comparative genomics: integrating genome structure, parasite biology and anthelmintic discovery

\author{
Martin T. Swain \\ Aberystwyth University \\ Denis M. Larkin \\ Aberystwyth University \\ Conor R. Caffrey \\ University of California - San Francisco \\ Stephen J. Davies \\ Uniformed Services University of the Health Sciences \\ Alex Loukas \\ James Cook University \\ See next page for additional authors
}

Follow this and additional works at: https://digitalcommons.unl.edu/usuhs

Part of the Medicine and Health Sciences Commons

Swain, Martin T.; Larkin, Denis M.; Caffrey, Conor R.; Davies, Stephen J.; Loukas, Alex; Skelly, Patrick J.; and Hoffmann, Karl F., "Schistosoma comparative genomics: integrating genome structure, parasite biology and anthelmintic discovery" (2011). Uniformed Services University of the Health Sciences. 91.

https://digitalcommons.unl.edu/usuhs/91

This Article is brought to you for free and open access by the U.S. Department of Defense at DigitalCommons@University of Nebraska - Lincoln. It has been accepted for inclusion in Uniformed Services University of the Health Sciences by an authorized administrator of DigitalCommons@University of Nebraska Lincoln. 


\section{Authors}

Martin T. Swain, Denis M. Larkin, Conor R. Caffrey, Stephen J. Davies, Alex Loukas, Patrick J. Skelly, and Karl F. Hoffmann 


\title{
Schistosoma comparative genomics: integrating genome structure, parasite biology and anthelmintic discovery
}

\author{
Martin T. Swain ${ }^{1 *}$, Denis M. Larkin ${ }^{1 *}$, Conor R. Caffrey ${ }^{2}$, Stephen J. Davies ${ }^{3}$, \\ Alex Loukas ${ }^{4}$, Patrick J. Skelly ${ }^{5}$ and Karl F. Hoffmann ${ }^{1}$
}

\author{
${ }^{1}$ Institute of Biological, Environmental and Rural Sciences (IBERS), Aberystwyth University, Aberystwyth, UK \\ ${ }^{2}$ Sandler Centre for Drug Discovery, California Institute for Quantitative Biosciences (QB3), University of California San Francisco \\ (UCSF), San Francisco, CA, USA \\ ${ }^{3}$ Department of Microbiology and Immunology, Uniformed Services University of the Health Sciences, Bethesda, MD, USA \\ ${ }^{4}$ School of Public Health, Tropical Medicine and Rehabilitation Sciences, James Cook University, Cairns, Australia \\ ${ }^{5}$ Molecular Helminthology Laboratory, Division of Infectious Diseases, Department of Biomedical Sciences, Tufts University, \\ Cummings School of Veterinary Medicine, Grafton, MA, USA
}

\begin{abstract}
Schistosoma genomes provide a comprehensive resource for identifying the molecular processes that shape parasite evolution and for discovering novel chemotherapeutic or immunoprophylactic targets. Here, we demonstrate how intragenus and intergenus comparative genomics can be used to drive these investigations forward, illustrate the advantages and limitations of these approaches and review how post-genomic technologies offer complementary strategies for genome characterisation. Although sequencing and functional characterisation of other schistosome/platyhelminth genomes continues to expedite anthelmintic discovery, we contend that future priorities should equally focus on improving assembly quality, and chromosomal assignment, of existing schistosome/platyhelminth genomes.
\end{abstract}

\section{Comparative genome basics}

July 2009 marked a seminal date in the history of parasite genomics, helminthology and evolutionary biology. After more than 20 years of collaborative research, both Schistosoma japonicum [1] and Schistosoma mansoni [2] draft genomes were elucidated using whole-genome shotgun sequencing (see Glossary). Along with Schistosoma haematobium, these parasitic trematodes are responsible for most cases of human hepatosplenic (S. japonicum and S. mansoni) and urinary (S. haematobium) schistosomiasis [3], a chronic and morbid neglected tropical disease afflicting hundreds of millions of people in sub-Saharan Africa, Asia and South America [4]. Information contained in these genomes has fuelled optimism that novel drug targets, vaccine candidates and immunomodulatory gene products will be found leading to the development of urgently needed control strategies $[5,6]$.

However, to comprehensively interrogate this vast amount of sequence information, comparative genome investigations are necessary. Comparative genomics can

Corresponding author: Hoffmann, K.F. (krh@aber.ac.uk).

Authors contributed equally to the de novo intragenus comparative genomic analysis reported here.

\section{Glossary}

Acoelomate: schistosomes lack a coelom (an internal fluid filled body cavity) and instead, are comprised of a solid, triploblastic (ectoderm, mesoderm and endoderm), bilaterally symmetrical, body plan. Owing to the acoelomate (lacking a coelom) nature, schistosome organs develop in direct contact with these triploblastic tissues and not in fluid filled cavities.

BAC (bacterial artificial chromosome): a DNA construct used in whole-genome, shotgun sequencing projects that can be propagated through Escherichia coli. Genomic DNA insert sizes of $150-350 \mathrm{~kb}$ are typically found within BACs. BAC DNA constructs (found in Sm1 and CHORI 103 BAC libraries) were used to map genome scaffolds to $S$. mansoni chromosomes via FISH.

Comparative genomics: an informatics based technique used to compare the DNA sequences that comprise draft or finished genomes of organisms within the same phylum or across different phyla. By identifying similarities (homologous synteny blocks, HSBs) and differences (evolutionary breakpoint regions, EBRs) across genomes, schistosome comparative genomics attempts to: (i) provide insight into the processes that shape species evolution, (ii) identify essential genes suitable for chemotherapy development and (iii) predict potential immunoprophylactic gene products. For this review, intragenus (comparing draft S. mansoni and S. japonicum genomes) and intergenus (contrasting Schistosoma genomes to comparator genomes) comparative genomics analyses are discussed.

Contig: a contiguous sequence of DNA assembled from overlapping cloned DNA fragments. The $S$. mansoni genome was assembled from 50376 contigs and the $S$. japonicum genome was assembled from 95265 contigs.

Evolutionary breakpoint regions (EBRs): a non-aligning, genomic interval found between two adjacent HSB boundaries. These can comprise interchromosomal or intrachromosomal rearrangements. At $100 \mathrm{~kb}$ resolution $52 \mathrm{EBRs}$ were found, whereas at $10 \mathrm{~kb}$ resolution 477 EBRs were uncovered.

FISH (fluorescence in situ hybridisation): an experimental technique by which genome scaffolds are physically mapped to chromosomes and visualised by fluorescence microscopy. Upon publication, $43 \%$ of the $S$. mansoni genome assembly was unambiguously assigned to the seven autosomes and the ZW sex-determining pairs. A total of $81 \%$ of the $S$. mansoni genome assembly (version 5.0) has now been physically mapped to the karyotype.

Gap: a portion in the scaffold that is linked to two sequence verified contigs but has not been sequenced.

Gene ontology (GO): a standardised vocabulary of gene product attributes that is species neutral and applicable to both prokaryotes and eukaryotes. See http:// www.geneontology.org/ for more information.

Homologous synteny blocks (HSBs): a minimum of two adjacent markers in the same chromosome/scaffold between S. mansoni and S. japonicum genomes that share the same order in both species without interruption by an HSB from a different region of the same chromosome/scaffold or from a different chromosome/scaffold. In this preliminary intra-Schistosoma comparative genome analysis, HSBs of $100 \mathrm{~kb}$ and $10 \mathrm{~kb}$ were considered.

Interchromosomal rearrangements: an intraspecies genomic rearrangement that takes place between different Schistosoma chromosomes. At $100 \mathrm{~kb}$ resolution 10 interchromosomal rearrangements were found, whereas at $10 \mathrm{~kb}$ resolution 67 EBRs were uncovered. 
Intrachromosomal rearrangements: an intraspecies genomic rearrangement that occurs within the same Schistosoma chromosome. At $100 \mathrm{~kb}$ resolution 42 intrachromosomal rearrangements were found, whereas at $10 \mathrm{~kb}$ resolution 410 intrachromosomal rearrangements were uncovered.

Resolution: the length threshold (in bps) that defines a HSB. For $100 \mathrm{~kb}$ resolution, all HSBs shorter than $100 \mathrm{~kb}$ are excluded from further analyses. Likewise, for $10 \mathrm{~kb}$ resolution, all HSBs shorter than $10 \mathrm{~kb}$ are excluded from further analyses.

Scaffold: a reconstructed portion of the genome made by assembling overlapping contigs and gaps. The $50376 \mathrm{~S}$. mansoni contigs were assembled into 5745 scaffolds and the 95265 S. japonicum contigs were assembled into 25048 scaffolds. These scaffolds comprised the draft genome sequences of each schistosome species.

Schistosomulum: a schistosome lifecycle stage that develops immediately after cercarial penetration of the definitive vertebrate host. Characteristic features of this developmental form include a tegumental heptalaminate (seven membranes comprise two opposing trilaminte lipid bilayers) covering and an ability to initiate haematophagy (blood feeding). The schistosomulum is believed to be a major target of protective host immune responses [51].

Tegument: a protective syncytium layer sandwiched between the hepatolaminate surface covering and the acoelomate schistosome body plan. Nucleated cell bodies situated below the tegument produce the diverse biomolecules and vesicles that are transported throughout the syncytium. Owing to the positional (host-parasite interface) and protective nature of this structure [55], proteins shuttled from subtegumental cell bodies to the hepatolaminate membrane surface are of interest for vaccinologists.

Whole-genome shotgun sequencing: a genome sequencing strategy, by which fragmented genomic DNA (gDNA) is randomly sequenced, assembled into overlapping contiguous sequences (contigs) and built into large genome scaffolds. Scaffolds are subsequently assembled into whole draft genomes of the studied organism. Whole-genome shotgun sequencing of the $S$. mansoni genome ( $363 \mathrm{Mb}$ at $6 \mathrm{X}$ coverage) was initiated with mixed-sex cercariae gDNA whereas the $S$. japonicum genome ( $397 \mathrm{Mb}$ at $6 \mathrm{X}$ coverage) was elucidated by gDNA isolated from mixed-sex adult schistosomes.

Z/W sex-determination pair: the diploid schistosome genome is maintained across seven autosomal and one Z/W sex-determining chromosomal pairs. The gender of individual schistosomes is dependent upon inheritance of these Z/W sex-defining chromosomes. A female schistosome will, therefore, contain a ZW combination of sex-determining chromosomes, whereas a male schistosome will contain a ZZ combination. Owing to challenges with physical mapping of the S. mansoni assembly (version 5.0) to individual Z or W chromosomes, a concatenated Z/W description is indicated (in this review as well as in the $S$. mansoni genome description [2]). Further studies undoubtedly will facilitate physical mapping of the most recent genome assembly to specific $Z$ and $W$ chromosomal regions.

be defined on many levels, but for the purpose of this review, we will discuss how intergenus and intragenus genome analyses are shaping schistosome post-genomic activities. Intergenus analyses involve cross-phylum genome comparisons in the primary search for gene loss/gene gain events. Both schistosome genome reports employed intergenus comparative genomics by contrasting the Schistosoma genomes with other genomes to discover features specific to, or shared between, parasitic and nonparasitic organisms [1,2]. This approach is similar to that performed for other parasitic helminth genome projects (e.g. Brugia malayi [7] or Trichinella spiralis [8]) and has identified metabolic chokepoints, conserved druggable targets, expanded gene families and protein domain loss within the Schistosoma.

Intragenus comparative genome analyses involve the exploration of relatedness/differences between species (within a single genus), which in turn could provide physical markers of chromosomal evolution and highlight conserved regions (e.g. protein coding genes or gene families) of possible relevance as anti-schistosomal targets. For the apicomplexans [9] and trypanosomes [10], intragenus comparative genomics has already facilitated the discovery of novel biological observations (i.e. core genomes and lineage specific expansions) that may lead to innovative anti-protozoan treatments. To date, similar intragenus analyses have not been performed for schistosomes.

Here, for the first time, using the most recent Schistosoma genome assemblies (SJR2 for S. japonicum and SMA5.0 for $S$. mansoni), an intragenus comparative genomic analysis is performed. Utilising a proven strategy to compare phylogenetically related genomes [11], regions of chromosomal similarity and dissimilarity are identified between basal- (S. japonicum) and derived(S. mansoni) schistosome species [12]. Complementing this karyotypic analysis is a review of how improvements in intergenus comparative and functional genomics can be applied to identify schistosome gene products of potential importance to parasite viability, development and host interrelationships. We suggest that the collective application of these comparative/functional genomics methodologies will lead to a better understanding of schistosome genome structure, gene function and evolution.

Intragenus Schistosoma comparative genomic analyses For the 2009 S. mansoni genome assembly [2], 43\% (distributed over 153 scaffolds) was unambiguously mapped onto schistosome chromosomes (seven autosomes and the Z/W sex-determination pair). Mapping was achieved by fluorescence in situ hybridisation (FISH) of bacterial artificial chromosomes (BACs) containing parasite genomic DNA fragments [13]. In 2011, with improvements in genome reassembly and additional FISH experiments, $81 \%$ of the $S$. mansoni genome (comprising 885 scaffolds, version 5.0) can now be explicitly mapped to chromosomes (M. Berriman, unpublished) (Figure 1a). This robust physical map allowed us to align the $S$. japonicum whole genome assembly (Figure $1 \mathrm{~b}$ ) to $S$. mansoni chromosomes (Figure 1c) and subsequently generate a comparative genomic map (Figure 1d). Blocks of intragenus homologous synteny (at $100 \mathrm{~kb}$ and $10 \mathrm{~kb}$ minimum size resolutions) are illustrated with the corresponding $S$. japonicum protein coding genes contained within these regions described in Supplementary Tables S1 and S2. At $100 \mathrm{~kb}$ resolution, the homologous synteny blocks (HSBs) cover approximately a quarter to one-third of the Schistosoma genome (101.17 and $112.93 \mathrm{Mb}$ of the $S$. japonicum and $S$. mansoni genomes, respectively) and most probably represent regions of chromosomes derived from a common ancestor [14] (Figure 2), which may be shared in all Schistosoma species. In mammalian genomes, HSBs are enriched for genes involved in developmental processes, neurogenesis and cell-to-cell interaction [11]. Accordingly, in schistosomes, these HSBs (containing 4443 genes at $10 \mathrm{~kb}$ resolution, Supplementary Table S2) could encode products suitable for the identification of pan-species drug and vaccine targets (further discussed below). Our analysis shows that boundaries of adjacent HSBs found in the same $S$. japonicum scaffold tend to flank putative evolutionary breakpoint regions (EBRs) between $S$. mansoni and $S$. japonicum genomes. These EBRs can be derived either from large-scale interchromosomal (black arrows and numbers, Figure 2) or intrachromosomal (red arrows and numbers, Figure 2) rearrangements and are created from the non-allele joining of broken double-stranded DNA ends during meiosis [15]. In mammalian genomes, 
(a)

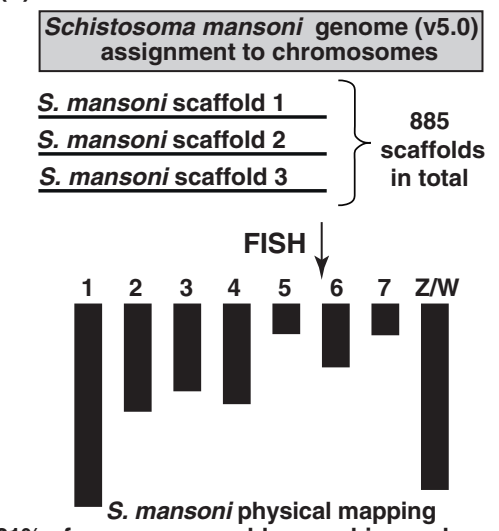

(b)

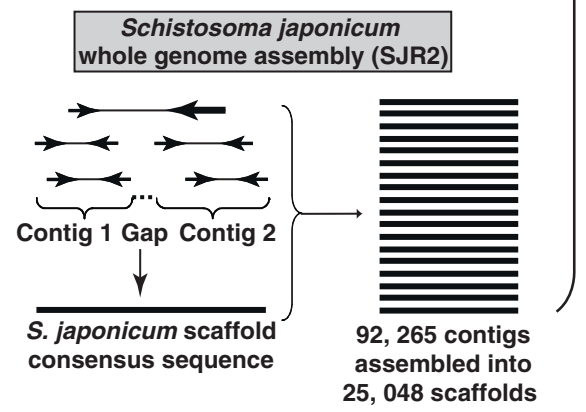

25,048 scaffolds (c)

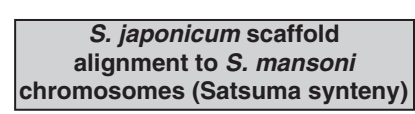

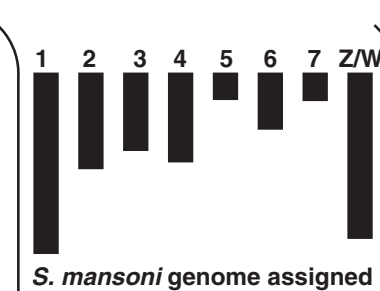
to chromosomes

(d)

\section{Virtual schistosome comparative \\ karyotype (Synteny tracker) \\ S. mansoni chromosomes}

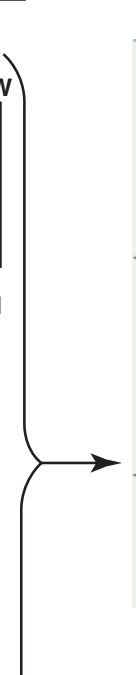

1
$=$
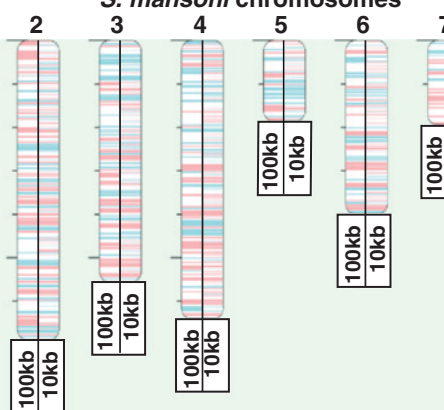

$7 \quad$ Z/W

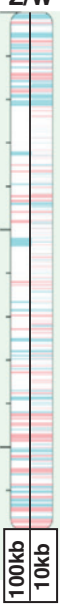

Visualised by Evolution Highway

(http://eh-demo.ncsa.uiuc.edu/)

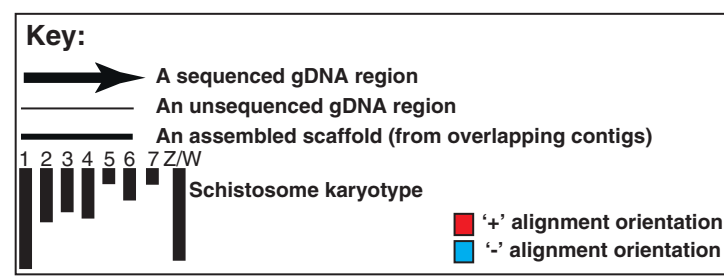

TRENDS in Parasitology

Figure 1. Intra-Schistosoma comparative genomic analysis: generation of a preliminary virtual karyotype. An intragenus comparative genome analysis was initiated with sequence information contained in the originally published S. japonicum genome (http://www.chgc.sh.cn/japonicum/Resources.html, SJR2) as well as the recently reassembled $S$. mansoni genome (http://www.sanger.ac.uk/resources/downloads/helminths/schistosoma-mansoni.html, SMA5.0). (a) Version 5.0 of the $S$. mansoni genome was reassembled into 885 scaffolds, where $81 \%$ of them were unambiguously assigned to chromosomal positions (seven autosomes and Z/W sex-determining chimera) by FISH (M. Berriman, unpublished). (b) The original S. japonicum genome assembly was assembled into 25048 scaffolds from 92265 contigs [1]. (c) To facilitate intergenus genomic comparisons, $S$. japonicum scaffolds were aligned to $S$. mansoni chromosomes by the Satsuma Synteny program [91] followed by detection of $>100 \mathrm{~kb}$ (full details found in Supplementary Table S1) and $>10 \mathrm{~kb}$ (full details found in Supplementary Table S2) homologous synteny blocks (HSBs) defined by the SyntenyTracker program [92]. (d) Both $100 \mathrm{~kb}$ and $10 \mathrm{~kb}$ HSB sets are visualised on the virtual S. mansoni comparative chromosomal map (generated by Evolution Highway, http://eh-demo.ncsa.uiuc.edu/). We used the $100 \mathrm{~kb}$ HSB set for identifying interchromosomal evolutionary breakpoint regions (EBRs) between schistosome genomes (Figure 2), whereas the $10 \mathrm{~kb}$ HSB set was used to interrogate gene ontology (GO) enrichment in all putative EBRs found between S. mansoni and S. japonicum genomes (Figure 3).

EBRs contain gene dense-, transcriptionally active-, poorly methylated- and replication initiation-rich elements $[16,17]$. These particular features are all associated with chromatin in a relaxed or open state and probably explain why evolutionary breakpoints occur here. As such, EBRs (and the genes contained within them) are subjected to greater genomic instabilities during normal cellular processes and are under increased evolutionary pressure to mutate [18].

Whereas all schistosome chromosomes, except chromosome 7, contain HSBs interrupted by interchromosomal EBRs at $100 \mathrm{~kb}$ resolution, closer interrogation of these EBRs (Figure 2, Supplementary Tables S1 and S2) revealed that Schistosoma genomes evolve preferentially by intrachromosomal rather than interchromosomal rearrangements (42 intrachromosomal EBRs vs 10 interchromosomal EBRs). This is consistent with nematode genomes [7,19] and illustrates a mechanism of chromosome evolution conserved between worm phyla. Furthermore, a ninefold increase in the total number of all genome rearrangements was found when the $10 \mathrm{~kb}$ set (477 EBRs comprising 410 intrachromosomal rearrangements and 67 interchromosomal rearrangements) was compared to the $100 \mathrm{~kb}$ set (52 EBRs). Numerical differences may indicate that: (i) a significant number of rearrangements missing in the $100 \mathrm{~kb}$ set are due to the scattered nature of the $S$. japonicum genome, (ii) errors in scaffold-to-chromosome assignments exist in one or both genomes and (iii) true small-scale synteny differences in $S$. mansoni and $S$. japonicum genomes are prevalent. We anticipate that resolution of these possibilities will be facilitated in the near future by advanced, intra-Schistosoma comparative genomics.

Although we have yet to fully detail the molecular features (e.g. DNA methylation status [20]) found within these Schistosoma EBRs, long terminal repeat (LTR) and non-LTR retrotransposable elements are found in a significantly greater proportion here (Supplementary Table S3). As these elements drive evolutionary processes [21] and have been associated with EBRs of mammalian genomes [22], their enrichment in schistosome EBRs is not surprising. The extent and role of these (functional or recently active) LTR and non-LTR retrotransposons in shaping schistosome evolution has yet to be determined, but is 


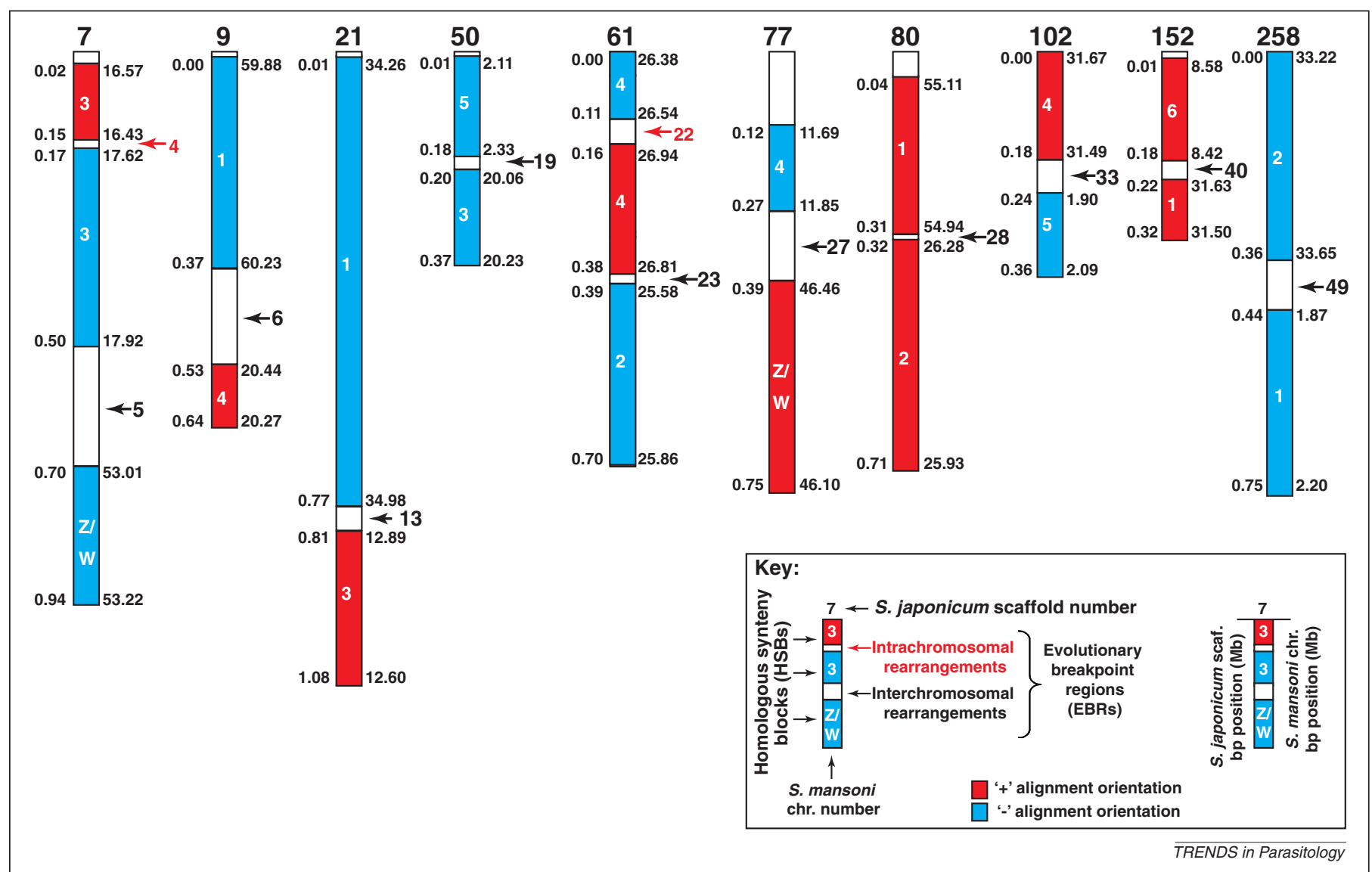

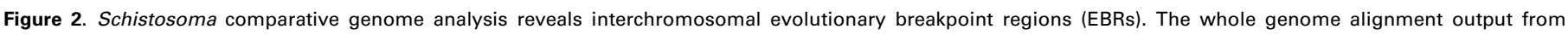
Satsuma Synteny [91] was translated into homologous synteny blocks (HSBs, $>100 \mathrm{~kb}$ ) using the SyntenyTracker program [92]. Combined, these HSBs (red blocks, ' + ' alignment orientation; blue blocks, '-' alignment orientation) cover $112.93 \mathrm{Mb}(31 \%)$ of the S. mansoni genomes and $101.17 \mathrm{Mb}$ ( $25 \%$ ) of the S. japonicum genomes,

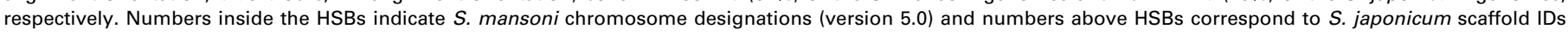
(SJR2). Numbers (indicated as Mb ranges) to the right side of the HSBs are $S$. mansoni chromosomal positions and the numbers to the left side of the HSBs are coordinates

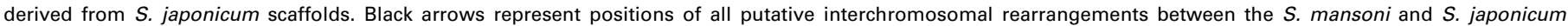

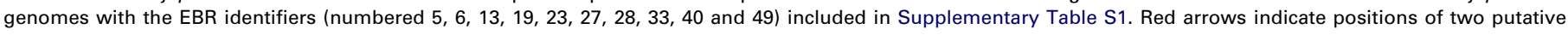
intrachromosomal rearrangements (out of 42 identified in this analysis) between the $S$. mansoni and $S$. japonicum genomes. Details of all intrachromosomal rearrangements can also be found in Supplementary Table S1.

an area of obvious interest. Further gene ontology (GO) analysis (Supplementary Table S2) suggests that, similar to mammalian EBRs, these parasite genomic features are gene-rich and, therefore, probably composed of open chromatin. This conclusion is supported by the finding that 'cell part' and 'catalytic activity' GO categories, belonging to the 'cellular component' and 'molecular function' high level GO terms (populated by gene products involved in transcription and DNA replication/repair), are significantly enriched within the Schistosoma EBRs (Figure 3 and Supplementary Table S2). It is tempting to speculate that lineage-specific $S$. mansoni or $S$. japonicum markers are also found within these EBRs and may be mined from within the 140 and 854 gene products identified at $100 \mathrm{~kb}$ (Supplementary Table S1) and $10 \mathrm{~kb}$ resolution (Supplementary Table $\mathrm{S} 2$ ), respectively.

Although this analysis has detected a core set of HSBs containing $\sim 25 \%$ of the ancestral Schistosoma genome, we expect that the number of EBRs in the two genomes is underestimated because a comparable physical $S$. japonicum genome map is not yet available. Progress in intra-Schistosoma comparative genomics will depend on improving genome assemblies mapped to chromosomes (e.g. S. japonicum) and de novo sequencing and draft assemblies of other schistosome genomes (e.g. S. haematobium). Indeed, recent intragenus comparative genome analysis of Caenorhabditis elegans and Caenorhabditis briggsae have improved defective gene models, detected potential new genes and identified missing orthologous relationships within this free-living nematode genus [23]. Further iterations of the present analysis will help identify molecular drivers of schistosome evolution (e.g. functional retrotransposable elements within EBRs) and highlight conserved regions (e.g. protein encoding genes or gene families within HSBs) of interest as pan-specific, anti-schistosomal targets. Comparative genomics between the Platyhelminthes will also be possible in the near future due to the completion or ongoing sequencing of Schmidtea mediterranea [24], Taenia solium [25], Echinococcus sp. and Hymenolepsis microstoma genomes (sequencing/draft assembly in progress for the last two helminths at the Wellcome Trust Sanger Institute). These efforts will help us to understand the origin and nature of parasitism within the phylum as well as highlight the chromosomal events associated with the evolution and divergence of parasitic trematodes and cestodes from free-living turbellarians. 


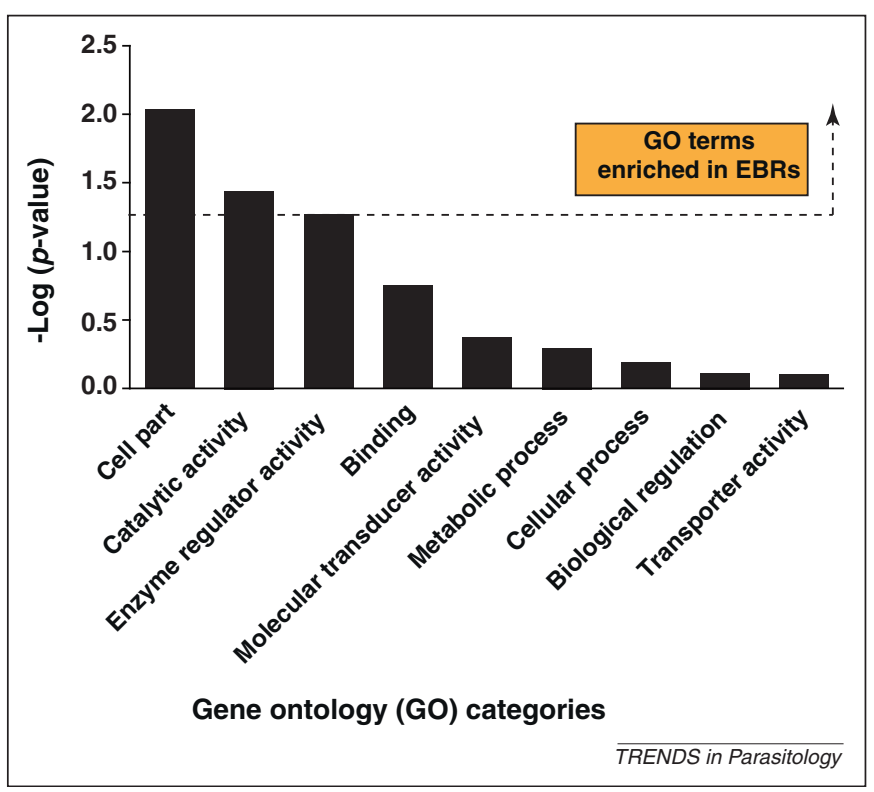

Figure 3. Schistosome evolutionary breakpoint regions (EBRs) and surrounding intervals $(<10 \mathrm{~kb})$ are enriched for specific gene ontology (GO) categories. GO categories (across molecular function, biological process and cellular component terms) were assigned to $S$. japonicum genes using an in-house script that attempted to assign gene products to GO names and synonyms from version 1.2 (release date 15 March, 2011 CVS revision number 1.1836) of the ontology (http:// www.geneontology.org). The script was able to calculate a score for each GO ID by matching words in the gene product to words in the GO name or synonym. Gene products that scored above a minimum score (or confidence value) were allocated to their highest scoring GO ID. The script then followed the links through the GO hierarchy to identify the corresponding top-level GO categories for each allocated GO ID. These top-level GO categories were subsequently used in the analysis. In the case of a gene product for which no GO name or synonym scored above the confidence value, no GO category was assigned. S. japonicum scaffolds were divided into $10 \mathrm{~kb}$ windows and the number of genes from each GO category that had $>100$ assigned genes was counted in each $10 \mathrm{~kb}$ window. Next, the average number of genes for each GO category was calculated separately for the windows located within EBRs and the remainder of $S$. japonicum scaffolds. The average number of genes was calculated for $10 \mathrm{~kb}$ windows located within the EBRs and compared with the average number of genes in $10 \mathrm{~kb}$ windows found outside the EBRs using a $t$-test with unequal variances as described previously [93]. The significance threshold $(P=0.05)$ is indicated by a horizontal dashed line. All GO categories above this threshold are more likely to occur in EBRs when compared to the rest of the genome, whereas GO categories below this threshold do not demonstrate any preferential localisation in EBRs compared with other regions of the genome.

\section{Intergenus comparative genomics to predict Schistosoma drug targets}

A major goal consequent on the arrival [1,2] and continuing refinement [26] of annotated schistosome genomes is the identification of new drug targets. In this regard, intergenus comparative genomics implements a series of user-defined criteria to derive short lists of prioritised and actionable gene targets [27-30]. Short-listed, highquality targets are derived from sequence comparisons between schistosome(s) and comparator(s) genomes to identify genes for which experimental data indicate that their products are essential to parasite survival or viability. Actionable entails having the experimental tools at hand to interrogate short-listed targets. So far, target validation in schistosomes has predominantly used transient RNA interference (detailed below) or more recently, employing vector-based small hairpin RNAs (shRNAs) $[31,32]$. The alternative or complementary approach of chemical validation relies on small molecules most often developed against a distinct (usually human) target with the implication, and sometimes formal demonstration $[33,34]$, that the anti-schistosomal effect is associated with binding to the intended schistosome orthologue(s). Based on the presented intra-Schistosoma comparative genomic analysis, an additional criterion for short-list inclusion and target validation would be chromosomal position. For example, 252 eukaryotic protein kinases (ePKs) have recently been identified in $S$. mansoni and many of these may be potential drug targets [35]. Our analysis (Figures 1 and 2) indicates that some ePKs (e.g. Sjp_0015710.1, Sjp_0019720.1, Sjp_0027360.1, Sjp_0056860.1) are physically positioned within intra-schistosomal EBRs (Supplementary Table S2). As EBRs are actively evolving, the selection of pan-Schistosoma chemotherapeutic targets physically positioned within these regions should proceed cautiously.

Online tools are under development to utilise comparative genomics for short-listing possible drug targets. For example, the TDR Targets Database (http://tdrtargets.org/) provides a variety of 'tunable' and Boolean-capable filters to generate user-defined lists of potential targets for 11 different pathogens, including $S$. mansoni and several other helminths [29,30]. In addition, SchistoDB (http:// schistodb.net/) [36] will expand considerably in the next year with improved query functionality, graphical user interfaces and other features from the EuPathDB family of genome databases [37] (G. Oliveira, L. Wei, J. Kissinger and D. Roos, personal communications). Utilising these resources, intergenus comparative genomics can be used to identify pathogen-specific proteins or those that are sufficiently different so as to be functionally absent from the host [38]. This is in an attempt to limit potential drug toxicity arising from chemical crossreactivity between the orthologous parasite and host targets. Yet, the alternative (i.e. to explore orthologous genes as a source of potential drug targets) is also useful. Here, genes that are shared among species are likely to be essential and thus more attractive as targets due to the severity of phenotypes produced upon perturbation [38,39]. Sufficient physiological, parasitological and/or protein structural circumstances often exist that can offset possible host toxicity [28] and, therefore, this concept has been actively exploited in recent comparative genomic studies involving $S$. mansoni [28,29]. This approach might also focus on those genes that are expressed in both juvenile and adult worms [40-42] considering that the current schistosomicide, praziquantel, is less effective against immature parasites [43].

Although comparative genomics offers a straightforward strategy to prevalidate potential drug targets, there are limitations, both inherent and contextual. For the latter, perhaps most obvious is the dearth of comprehensive genome, transcriptome and proteome information for $S$. haematobium which is more prevalent than $S$. mansoni in Africa and is often co-endemic [44]. With the current operational requirement in sub-Saharan Africa for a single anti-schistosomal drug, this lack of annotated sequence information represents a crucial knowledge gap only partially filled by the 0.5 to $1.0 \mathrm{x}$ coverage genome sequence that is currently available (http://www.cebio.org/projetos/ schistosoma-haematobium-genome). Even if highly processed/assembled S. haematobium genomic data were at 
hand, it might be difficult to predict how even subtle sequence differences between $S$. mansoni and $S$. haematobium targets would impact the successful development of a single drug. Also, as comparative genomics incorporates experimental loss-of-function data from model organisms, the approach may miss potential targets modulated through gain-of-function, as occurs with many of today's anthelmintics [28]. Looking much further forward, comparative genomics and the underlying (but increasingly challenged) drug discovery philosophy of 'one gene, one target, one disease' [45] may oversimplify the process of identifying new drug targets. Rather, a better understanding of the dynamics of how biological pathways and networks are perturbed by small molecules (polypharmacology) and/or gene disruption would provide the sophistication necessary to predict new therapeutic targets [45-47], especially with a view to limiting the establishment of drug resistance. Finally, based on the findings presented here, selecting compounds that target loci contained within HSBs (Figure 2, Supplementary Tables S1 and S2) presents new opportunities to identify therapeutic small molecules.

\section{Comparative schistosome vaccinomics/immunomics: identifying immunoprophylactic targets within the tegument}

Apart from potential drug targets, the schistosome genome $[1,2]$, various transcriptomes [48,49] and proteomes of the tegument and excretory/secretory (ES) products (reviewed in [50]) have also provided researchers with a 'molecular haystack' in which to find a handful of 'immunoprophylactic needles'. A major challenge is to efficiently and comparatively mine this information. DNA microarrays have partially addressed this issue with several recent reports [40,41] identifying gene products differentially expressed throughout the lifecycle of the parasite, including the

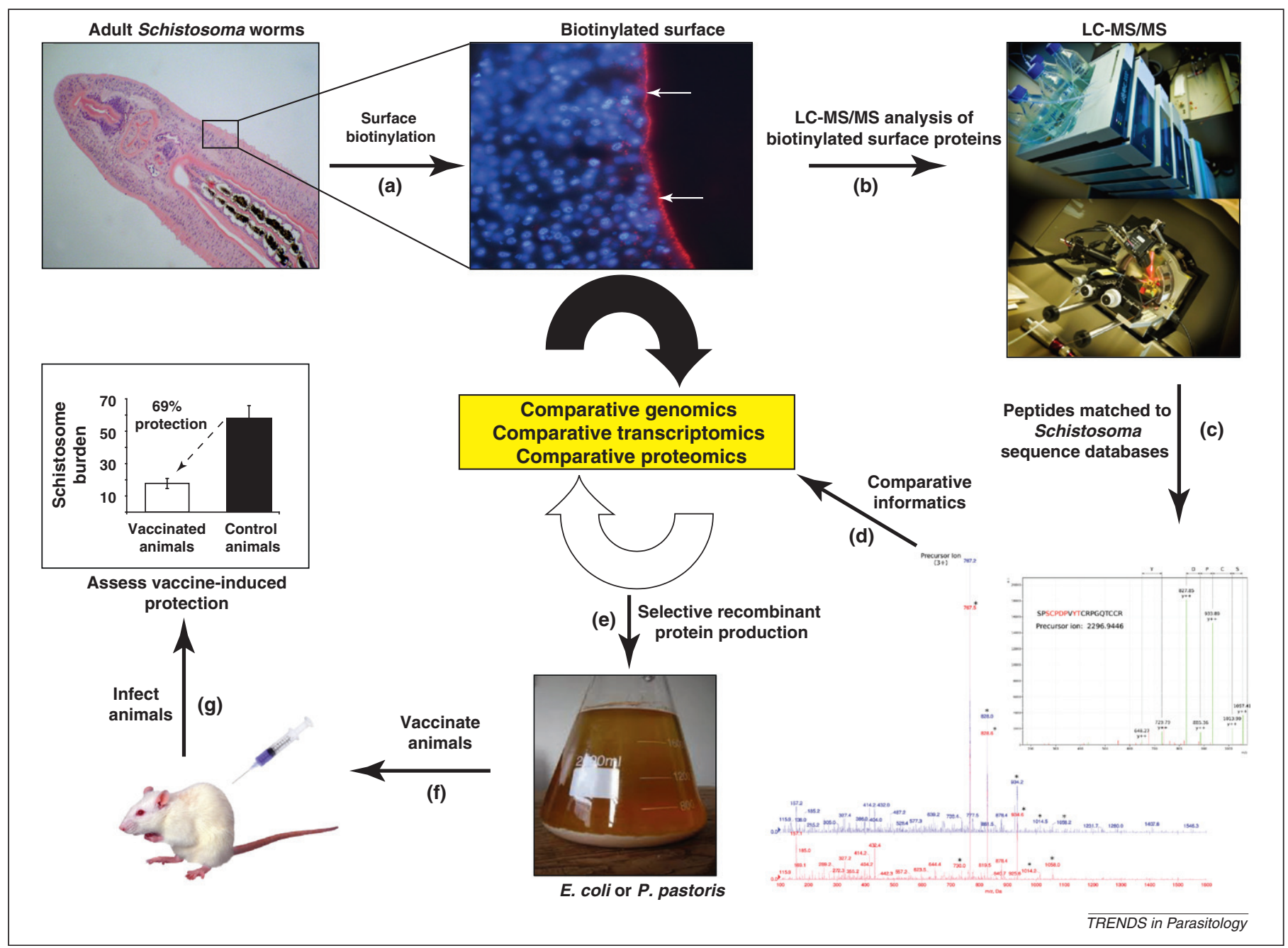

Figure 4. Comparative informatics approaches for identifying vaccine candidates from the schistosome tegument. Owing to advances in 'omics' technologies, next-generation schistosome vaccine discovery has been revitalised. Outlined is an example of how complementary strategies are currently being used to identify next-generation, antischistosomal vaccine targets. (a) To identify tegument proteins exposed at the host-parasite interface, schistosome worms (adults represented here) are labelled with biotin [60] [tegument visualised here by incubating adult worms with Cy3-labelled anti-biotin Abs (red sinuous band indicated by white arrows) and co-stained with DAPI to distinguish subtegumental nuclei (blue)]. (b) Subsequent processing of these biotin-labelled tegumental preparations generates a collection of putative, surface-exposed proteins that can be analysed by Liquid chromatography-mass spectrometry/mass spectrometry (LC-MS/MS). (c) Peptide spectra generated from MS/MS analysis are matched to gene/cDNA sequences from Schistosoma databases, allowing for the identification of parasite proteins. (d) Protein sequences are filtered through a cyclic round of informatics involving comparative genomics (protein sequences found only in the Platyhelminthes or also present in other genomes), comparative transcriptomics (intra-Schistosoma gene expression similarities throughout the parasite lifecycle [40,41]) and comparative proteomics (proteins found in tegumental preparations across the Schistosoma [59-61]). (e) Selected recombinant proteins that display desirable features (i.e. expressed in schistosomula as well as adults and are surface exposed in the Schistosoma teguments) are produced in a heterologous expression system (e.g. Escherichia coli or Pichia pastoris), purified and used to (f) vaccinate laboratory animals to (g) assess protective efficacy. 
tissue migrating schistosomulum - a major target of protective immune responses [51]. As many of these transcripts (e.g. tetraspanins, cathepsins, serpins and tegumental-associated antigens) are encoded in both $S$. mansoni and $S$. japonicum genomes (i.e. found within HSBs, Supplementary Table S2) and are also expressed in all definitive host lifecycle stages, they represent key vaccine candidates.

Given the acoelomate body plan of the parasite, schistosome tissues and organs cannot easily be manually dissected to probe for orthologous gene products (identified by comparative genomics) of possible relevance as vaccine antigens. To overcome this challenge, laser microdissection microscopy (LMM) has helped to elucidate gene expression in specific tissues of both $S$. mansoni and $S$. japonicum. Thus far, the gastrodermis and reproductive tissues have been scrutinised using a combination of LMM, RNA extraction and DNA microarray analysis $[52,53]$. This novel comparative transcriptomics approach aided the assembly of a schistosome gene atlas for the organs under study [53]. Although this methodology offers an unprecedented opportunity to identify tissue-enriched transcriptional profiles (between species), it has its limitations. For example, the schistosome tegument, a tissue often targeted by vaccinologists [54] (being situated at the host-parasite interface [55]) does not readily lend itself to LMM. This is because the tegumental cell bodies (and their nuclei) are buried deeply within the musculature.

Alternative approaches to 'dissect' the tegument have utilised comparative proteomics to discover and characterise proteins of interest at this host-parasite interface [50,56-60]. This approach has revealed that several tegumental proteins are homologous between schistosome species $[56,59,60]$ with some being localised to the outer, host-interacting, surface membrane [60,61]. It is these surface-exposed proteins that are attractive vaccine targets (Figure 4). For example, many tetraspanin genes (contained within the S. japonicum and S. mansoni HSBs, Figure 1 and Supplementary Table S2) are highly expressed in the intra-mammalian schistosome stages $[40,41]$, and some of these are located in tegumental membranes $[60,61]$. The tetraspanins have been experimentally proven to be protective in small and large animal models of schistosomiasis [62,63] and Sm-TSP-2 is also selectively recognised by naturally resistant humans [54,62]. Thus, (comparative) proteomics has been a successful platform in the identification and short-listing of candidate protein vaccines.

Integration of 'omics' technologies has accelerated the development of new post-genomic tools to aid vaccine discovery. For example, high-throughput protein expression techniques (e.g. in vitro transcription/translation systems), coupled to high-throughput immunoscreening with sera from resistant humans and animals, have propelled schistosomiasis research into the immunomics era [64]. Recently, the first Schistosoma immunomics protein microarray was described as a vaccine discovery tool [65]. Antigens included on this microarray were chosen from those identified in previous proteomic investigations in addition to those sequences selected from comparative in silico screening of both $S$. mansoni and $S$. japonicum genomes and transcriptomes. The goal here was to identify target antigens based on subcellular location with a particular emphasis on those proteins expressed in the tegument. Proteins were expressed in a cell-free in vitro transcription/ translation system and contact-printed onto nitrocellulosecoated slides to form protein microarrays. The arrays are currently being probed with IgG and IgE antibody subclasses from resistant and chronically infected human and animal populations (S. Gaze and A. Loukas, unpublished). This approach is revealing antigens that are targets of both protective IgG responses and potentially harmful $\operatorname{IgE}$ responses [66]. This innovative technology of immunomics or, essentially, reverse vaccinology that relies on the outputs of comparative genomics, transcriptomics and proteomics analyses has the potential to transform vaccine research for schistosomiasis and other parasitic diseases.

\section{Functionally exploring comparative genomic outputs: revisiting the tegument}

Identifying the precise role(s) of short-listed chemotherapeutic or immunoprophylactic targets is often problematic, especially for those genes without orthology outside of the genus or that encode proteins of unknown function [1,2]. For schistosomes, RNAi has emerged as a useful technique to experimentally manipulate the expression of specific schistosome genes and, possibly, gain insight into gene function. Newer protocols employing shRNAs may further extend its utility [31,32]. Comparative schistosome genomics has revealed that genes encoding known RNAi pathway effectors are shared between $S$. mansoni and $S$. japonicum $[67,68]$. Furthermore, targeted gene suppression (mediated by RNAi) has been demonstrated in both species $[69,70]$. Although RNAi can be both rapid and longlasting [71,72] as well as functional in several schistosome lifecycle stages [71,73], it is not always definitive. For reasons unknown, some genes appear recalcitrant to robust suppression [72,74], and striking phenotypes are seldom seen [72,73]. In other cases, RNAi has helped identify drug targets and gain insight into parasite biochemistry, physiology and host-parasite interactions $[34,70,75,76]$.

Perhaps due to the voluminous genome data, schistosome functional genomics has most often focused on a conserved biochemical pathway, tissue type or employed a gene-by-gene approach. As an example, we review how RNAi has contributed to the functional characterisation of conserved (within the Schistosoma) proteins in one such tissue, the aforementioned tegument. Part of its functioning at the host-parasite interface is to import nutrients from the host bloodstream. Within the $S$. mansoni tegument, two facilitated diffusion, glucose-importing proteins have been identified; schistosome glucose transporter (SGTP) 1 and SGTP4 [77]. SGTP4 is present in the host interactive, apical tegumental membranes, whereas SGTP1 is found in the tegumental basal membrane and other tissues. Using schistosomula and adult worms, RNAi of SGTP1 or SGTP4 impaired the ability of the parasite to import glucose, and this effect was compounded by suppression of both transporter genes simultaneously [78]. Although RNAi-treated parasites cultured in rich medium were not phenotypically different from controls as assessed 
microscopically, their survival in vivo was significantly impaired [78]. Thus, RNAi has provided direct evidence for the importance of SGTP1 and SGTP4 in glucose transport and survival in the mammalian host.

Proteomic analysis of schistosome tegumental membranes also revealed the presence of an aquaporin homolog (SmAQP) [56]. Aquaporins (found within HSBs, Figure 1 and Supplementary Table S2) are membrane proteins that form pores to selectively conduct water molecules into and out of cells. RNAi-mediated SmAQP suppression impaired the ability of schistosomula to osmoregulate and revealed the previously unrecognised role of the tegument in controlling water movement into, and out of, the parasites [79]. Unexpectedly, SmAQP-suppressed adult parasites in vitro failed to rapidly acidify their culture medium and were found to excrete less lactate compared with controls [80]. Heterologous expression of SmAQP in Xenopus oocytes demonstrated that this protein, in addition to transporting water, could also transport lactate [80]. These findings provide a molecular understanding of how schistosomes cope with the significant quantities of lactate created from the largely anaerobic glucose catabolism that is a hallmark of their intravascular lives [81]. Collectively, the comparative and functional genomic data suggest that the syntenic position of aquaporins is an important feature of schistosome/host relationships driven by the need to extend tegumental functions in metabolic waste excretion.

The contribution of the tegument to immune evasion by the worms is less well understood, although many investigations have provided functional explanations in this regard [55]. The presence of nucleotide-metabolizing enzymes associated with the parasite surface $[56,57]$ has led to the suggestion that these can catabolise host proinflammatory metabolites such as ATP and generate anti-inflammatory mediators such as adenosine [82]. Accordingly, schistosomes may prevent their hosts from focusing immunological mediators in their vicinity [82]. In support of this notion, RNAi of adult tegumental alkaline phosphatase (SmAP) prevented production of the antiinflammatory adenosine from an exogenously added precursor (AMP) [83].

These examples illustrate the power of RNAi to provide insight into schistosome gene function. Combined with schistosome transcriptomics and proteomics, RNAi use can be extended to systematically identify the function of many comparatively identified genome products in parasite biology, development and host interactions.

\section{Concluding remarks and future perspectives}

As outlined, comparative genomics offers an established set of principles for understanding gene and genome biology as well as exploiting that information to identify targets for chemotherapy and immunotherapy. The intragenus and intergenus comparative genomic analyses discussed here are an encouraging first step, but will be improved (Box 1) once other schistosome genomes are sequenced (e.g. $S$. haematobium) and existing schistosome genomes are refined, reassembled and mapped (e.g. S. japonicum). When

\section{Box 1. Outstanding questions: the future of schistosome comparative genomics}

\section{How important is genome reassembly?}

To maximise the power of comparative genomics, Schistosoma genomes need to be continually reassembled into the smallest set of overlapping scaffolds as possible. This allows for large stretches of multiple genomes to be systematically compared. Although the $S$. mansoni genome has undergone several rounds of reassembly (genome version 5.0 has 885 scaffolds; http://www.sanger.ac.uk/ resources/downloads/helminths/schistosoma-mansoni.html), there has been very little progress in reassembling the $S$. japonicum genome since publication (SJR2 has 25048 scaffolds; http:// www.chgc.sh.cn/japonicum/Resources.html). Therefore, our preliminary intragenus comparative genome analysis is limited by the fragmented nature of the $S$. japonicum genome. Future analyses will be improved upon refined and reassembled Schistosoma genomes facilitated by application of next generation sequencing technologies and sophisticated informatics. Assigning the reassembled $S$. japonicum genome to chromosomes (similar to that performed for $S$. mansoni [13]) would also aid future intragenus analyses.

Is the $\boldsymbol{S}$. haematobium genome important for future comparative analyses?

The preliminary Schistosoma comparative genomic analysis provided in this review would significantly benefit from the elucidation of the $S$. haematobium genome. A variety of evolutionary and control questions could be more readily addressed upon the inclusion of this most derived (amongst S. japonicum, S. mansoni and $S$. haematobium) schistosome species. Efforts are currently underway to sequence the genome of this species (e.g. http:// www.cebio.org/projetos/schistosoma-haematobium-genome). Also, comprehensive genome information for $S$. haematobium would be essential given the operational simplicity demanded for a single drug or vaccine to control both $S$. mansoni and $S$. haematobium in sub-Saharan Africa.

\section{How important are additional platyhelminth genomes?}

Comparative genomics of only two species in one class within the phylum (illustrated in this review) is obviously providing a very limited snapshot of platyhelminth biology. Therefore, the elucidation of new flatworm genomes is necessary to understand the evolution of this important group of animals. Genomes of Echinoccocus granulosus (http: //www.sanger.ac.uk/resources/downloads/helminths/echinococcusgranulosus.html), Echinoccocus multilocularis (http://www.sanger.ac.uk/ resources/downloads/helminths/echinococcus-multilocularis.html), Hymenolepsis microstoma (http://www.sanger.ac.uk/resources/ downloads/helminths/hymenolepis-microstoma.html), Schmidtea mediterranea (http://smedgd.neuro.utah.edu/) and Taenia solium are all at different states of assembly, but upon completion, will advance our ability to perform comparative flatworm genomics. Information contained within new platyhelminth transcriptomes [84-87] will also complement certain aspects of comparative genome investigations (i.e. identification of conserved drug or vaccine targets).

How can post-genomic technologies effectively complement platyhelminth comparative genomics investigations?

A large variety of post-genomics technologies (e.g. $[88,89])$ have been developed over the past decade to study platyhelminth biology. However, the optimism that these technologies would quickly deliver new platyhelminth drugs or vaccines has yet to be fully realised. Perhaps, in light of the enormous energy put forth in sequencing new platyhelminth genomes, equal emphasis should be dedicated towards the meta-analysis of existing post-genomics datasets to identify those conserved targets suitable for functional validation. Further refinement of post-genomic technologies applied to conserved targets identified by meta-analysis of available datasets and new targets identified by platyhelminth comparative genomics investigations will transform the state of anthelmintic discovery. Indeed, it is this type of approach that is beginning to make an impact on Plasmodium biology and the identification of novel antimalarials [90]. 
additional platyhelminths are included, a robust framework for comparative flatworm genomics will be realised. This information will lead to (i) detection of genes affected by the natural selection processes operating on each platyhelminth species, (ii) identification of the species- and class-specific gene network changes and (iii) characterisation of gene birth/death rates due to lineage-specific genome rearrangements, duplications and mobile genetic element activity. This greater knowledge of genome biology (experimentally verified by post-genomic technologies) will aid the discovery of novel schistosomiasis control strategies and allow for a better understanding of lifestyle diversity and evolution within the Platyhelminthes.

\section{Acknowledgments}

We thank the many individuals within and outside the global worm community who have supported and encouraged us throughout the years. These interactions have stimulated our inspection of the topics addressed in this review. We also acknowledge the Wellcome Trust, the US National Institutes of Health (AI-056273), the Australian National Health and Medical Research Council and the Sandler Foundation for financially supporting our research activities.

\section{Appendix A. Supplementary data}

Supplementary data associated with this article can be found, in the online version, at doi:10.1016/j.pt.2011. 09.003 .

\section{References}

1 Schistosoma japonicum Sequencing Consortium (2009) The Schistosoma japonicum genome reveals features of host-parasite interplay Nature 460, 345-351

2 Berriman, M. et al. (2009) The genome of the blood fluke Schistosoma mansoni. Nature 460, 352-358

3 Gryseels, B. et al. (2006) Human schistosomiasis. Lancet 368, 1106-1118

4 King, C.H. (2010) Parasites and poverty: the case of schistosomiasis. Acta Trop. 113, 95-104

5 Han, Z.G. et al. (2009) Schistosoma genomics: new perspectives on schistosome biology and host-parasite interaction. Annu. Rev. Genomics Hum. Genet. 10, 211-240

6 Webster, J.P. et al. (2010) Schistosome genomes: a wealth of information. Trends Parasitol. 26, 103-106

7 Ghedin, E. et al. (2007) Draft genome of the filarial nematode parasite Brugia malayi. Science 317, 1756-1760

8 Mitreva, M. et al. (2011) The draft genome of the parasitic nematode Trichinella spiralis. Nat. Genet. 43, 228-235

9 Cai, H. et al. (2010) Core genome components and lineage specific expansions in malaria parasites Plasmodium. BMC Genomics 11 (Suppl. 3), S13

10 El-Sayed, N.M. et al. (2005) Comparative genomics of trypanosomatid parasitic protozoa. Science 309, 404-409

11 Larkin, D.M. et al. (2009) Breakpoint regions and homologous synteny blocks in chromosomes have different evolutionary histories. Genome Res. 19, 770-777

12 Attwood, S.W. et al. (2007) A DNA sequence-based study of the Schistosoma indicum (Trematoda: Digenea) group: population phylogeny, taxonomy and historical biogeography. Parasitology 134, 2009-2020

13 Criscione, C.D. et al. (2009) Genomic linkage map of the human blood fluke Schistosoma mansoni. Genome Biol. 10, R71

14 Hardison, R.C. (2003) Comparative genomics. PLoS Biol. 1, E58

15 Sankoff, D. (2009) The where and wherefore of evolutionary breakpoints. J. Biol. 8, 66

$16 \mathrm{Ma}$, J. et al. (2006) Reconstructing contiguous regions of an ancestral genome. Genome Res. 16, 1557-1565

17 Lemaitre, C. et al. (2009) Analysis of fine-scale mammalian evolutionary breakpoints provides new insight into their relation to genome organisation. BMC Genomics 10, 335
18 Longhese, M.P. et al. (2006) The cellular response to chromosome breakage. Mol. Microbiol. 60, 1099-1108

19 Stein, L.D. et al. (2003) The genome sequence of Caenorhabditis briggsae: a platform for comparative genomics. PLoS Biol. 1, E45

20 Geyer, K.K. et al. (2011) Cytosine methylation regulates oviposition in the pathogenic blood fluke Schistosoma mansoni. Nat. Commun. 2, 424

21 Bohne, A. et al. (2008) Transposable elements as drivers of genomic and biological diversity in vertebrates. Chromosome Res. 16, 203-215

22 Elsik, C.G. et al. (2009) The genome sequence of taurine cattle: a window to ruminant biology and evolution. Science 324, 522-528

23 Vergara, I.A. and Chen, N. (2010) Large synteny blocks revealed between Caenorhabditis elegans and Caenorhabditis briggsae genomes using OrthoCluster. BMC Genomics 11, 516

24 Robb, S.M. et al. (2008) SmedGD: the Schmidtea mediterranea genome database. Nucleic Acids Res. 36, D599-D606

25 Aguilar-Diaz, H. et al. (2006) The genome project of Taenia solium. Parasitol. Int. 55 (Suppl.), S127-S130

26 Tsai, I.J. et al. (2010) Improving draft assemblies by iterative mapping and assembly of short reads to eliminate gaps. Genome Biol. 11, R41

27 Frearson, J.A. et al. (2007) Target assessment for antiparasitic drug discovery. Trends Parasitol. 23, 589-595

28 Caffrey, C.R. et al. (2009) A comparative chemogenomics strategy to predict potential drug targets in the metazoan pathogen, Schistosoma mansoni. PLoS ONE 4, e4413

29 Crowther, G.J. et al. (2010) Identification of attractive drug targets in neglected-disease pathogens using an in silico approach. PLoS Negl. Trop. Dis. 4, e804

30 Aguero, F. et al. (2008) Genomic-scale prioritization of drug targets: the TDR Targets database. Nat. Rev. Drug Discov. 7, 900-907

31 Zhao, Z.R. et al. (2008) Schistosoma japonicum: inhibition of Mago nashi gene expression by shRNA-mediated RNA interference. Exp. Parasitol. 119, 379-384

32 Ayuk, M.A. et al. (2011) Schistosoma mansoni U6 gene promoterdriven short hairpin RNA induces RNA interference in human fibrosarcoma cells and schistosomules. Int. J. Parasitol. 41, 783-789

33 Abdulla, M.H. et al. (2007) Schistosomiasis mansoni: novel chemotherapy using a cysteine protease inhibitor. PLoS Med. 4, e14

34 Kuntz, A.N. et al. (2007) Thioredoxin glutathione reductase from Schistosoma mansoni: an essential parasite enzyme and a key drug target. PLoS Med. 4, e206

35 Andrade, L.F. et al. (2011) Eukaryotic protein kinases (ePKs) of the helminth parasite Schistosoma mansoni. BMC Genomics 12, 215

36 Zerlotini, A. et al. (2009) SchistoDB: a Schistosoma mansoni genome resource. Nucleic Acids Res. 37, D579-D582

37 Aurrecoechea, C. et al. (2010) EuPathDB: a portal to eukaryotic pathogen databases. Nucleic Acids Res. 38, D415-D419

38 McCarter, J.P. (2004) Genomic filtering: an approach to discovering novel antiparasitics. Trends Parasitol. 20, 462-468

39 Doyle, M.A. et al. (2010) Drug target prediction and prioritization: using orthology to predict essentiality in parasite genomes. $B M C$ Genomics 11, 222

40 Fitzpatrick, J.M. et al. (2009) Anti-schistosomal intervention targets identified by lifecycle transcriptomic analyses. PLoS Negl. Trop. Dis. 3 , e543

41 Gobert, G.N. et al. (2009) Developmental gene expression profiles of the human pathogen Schistosoma japonicum. BMC Genomics 10, 128

42 Jolly, E.R. et al. (2007) Gene expression patterns during adaptation of a helminth parasite to different environmental niches. Genome Biol. 8, R65

43 Doenhoff, M.J. et al. (2009) Praziquantel: its use in control of schistosomiasis in sub-Saharan Africa and current research needs. Parasitology 136, 1825-1835

44 Rollinson, D. (2009) A wake up call for urinary schistosomiasis: reconciling research effort with public health importance. Parasitology 136, 1593-1610

45 Hopkins, A.L. (2008) Network pharmacology: the next paradigm in drug discovery. Nat. Chem. Biol. 4, 682-690

46 Carlson, S.M. and White, F.M. (2011) Using small molecules and chemical genetics to interrogate signaling networks. ACS Chem. Biol. 6, 75-85

47 Durrant, J.D. et al. (2010) A multidimensional strategy to detect polypharmacological targets in the absence of structural and sequence homology. PLoS Comput. Biol. 6, e1000648 
48 Verjovski-Almeida, S. et al. (2003) Transcriptome analysis of the acoelomate human parasite Schistosoma mansoni. Nat. Genet. 35, 148-157

49 Liu, F. et al. (2006) New perspectives on host-parasite interplay by comparative transcriptomic and proteomic analyses of Schistosoma japonicum. PLoS Pathog. 2, e29

50 DeMarco, R. and Verjovski-Almeida, S. (2009) Schistosomesproteomics studies for potential novel vaccines and drug targets. Drug. Discov. Today 14, 472-478

51 McManus, D.P. and Loukas, A. (2008) Current status of vaccines for schistosomiasis. Clin. Microbiol. Rev. 21, 225-242

52 Gobert, G.N. et al. (2009) Tissue specific profiling of females of Schistosoma japonicum by integrated laser microdissection microscopy and microarray analysis. PLoS Negl. Trop. Dis. 3, e469

53 Nawaratna, S.S. et al. (2011) Gene atlasing of digestive and reproductive tissues in Schistosoma mansoni. PLoS Negl. Trop. Dis. 5, e1043

54 Loukas, A. et al. (2007) Schistosome membrane proteins as vaccines. Int. J. Parasitol. 37, 257-263

55 Skelly, P.J. and Alan Wilson, R. (2006) Making sense of the schistosome surface. Adv. Parasitol. 63, 185-284

56 Braschi, S. et al. (2006) The tegument surface membranes of the human blood parasite Schistosoma mansoni: a proteomic analysis after differential extraction. Proteomics 6, 1471-1482

57 Castro-Borges, W. et al. (2011) Enzymatic shaving of the tegument surface of live schistosomes for proteomic analysis: a rational approach to select vaccine candidates. PLoS Negl. Trop. Dis. 5, e993

58 van Balkom, B.W. et al. (2005) Mass spectrometric analysis of the Schistosoma mansoni tegumental sub-proteome. J. Proteome Res. 4 , 958-966

59 Perez-Sanchez, R. et al. (2006) Proteomic analysis of the tegument and excretory-secretory products of adult Schistosoma bovis worms. Proteomics 6 (Suppl. 1), S226-S236

60 Mulvenna, J. et al. (2010) Exposed proteins of the Schistosoma japonicum tegument. Int. J. Parasitol. 40, 543-554

61 Braschi, S. and Wilson, R.A. (2006) Proteins exposed at the adult schistosome surface revealed by biotinylation. Mol. Cell. Proteomics $5,347-356$

62 Tran, M.H. et al. (2006) Tetraspanins on the surface of Schistosoma mansoni are protective antigens against schistosomiasis. Nat. Med. 12, 835-840

63 Da'dara, A.A. et al. (2008) DNA-based vaccines protect against zoonotic schistosomiasis in water buffalo. Vaccine 26, 3617-3625

64 Doolan, D.L. (2011) Plasmodium immunomics. Int. J. Parasitol. 41, 3-20

65 Driguez, P. et al. (2010) Schistosomiasis vaccine discovery using immunomics. Parasites Vectors 3,4

66 Hotez, P.J. et al. (2010) Developing vaccines to combat hookworm infection and intestinal schistosomiasis. Nat. Rev. Microbiol. 8, 814-826

67 Luo, R. et al. (2010) Analysis and characterization of the genes encoding the Dicer and Argonaute proteins of Schistosoma japonicum. Parasites Vectors 3, 90

68 Krautz-Peterson, G. and Skelly, P.J. (2008) Schistosoma mansoni: the dicer gene and its expression. Exp. Parasitol. 118, 122-128

69 Zou, X. et al. (2011) RNAi silencing of calcium-regulated heat-stable protein of $24 \mathrm{kDa}$ in Schistosoma japonicum affects parasite growth. Parasitol. Res. 108, 567-572

70 Rinaldi, G. et al. (2009) RNA interference targeting leucine aminopeptidase blocks hatching of Schistosoma mansoni eggs. Mol. Biochem. Parasitol. 167, 118-126

71 Krautz-Peterson, G. et al. (2007) Optimizing gene suppression in schistosomes using RNA interference. Mol. Biochem. Parasitol. 153, 194-202
72 Stefanic, S. et al. (2010) RNA interference in Schistosoma mansoni schistosomula: selectivity, sensitivity and operation for larger-scale screening. PLoS Negl. Trop. Dis. 4, e850

73 Mourao, M.M. et al. (2009) Phenotypic screen of early-developing larvae of the blood fluke, Schistosoma mansoni, using RNA interference. PLoS Negl. Trop. Dis. 3, e502

74 Krautz-Peterson, G. et al. (2009) RNA interference in schistosomes: machinery and methodology. Parasitology 137, 485-495

75 Swierczewski, B.E. and Davies, S.J. (2009) A schistosome cAMPdependent protein kinase catalytic subunit is essential for parasite viability. PLoS Negl. Trop. Dis. 3, e505

76 Tran, M.H. et al. (2010) Suppression of mRNAs encoding tegument tetraspanins from Schistosoma mansoni results in impaired tegument turnover. PLoS Pathog. 6, e1000840

77 Skelly, P.J. et al. (1994) Cloning, characterization, and functional expression of cDNAs encoding glucose transporter proteins from the human parasite Schistosoma mansoni. J. Biol. Chem. 269, 4247-4253

78 Krautz-Peterson, G. et al. (2010) Suppressing glucose transporter gene expression in schistosomes impairs parasite feeding and decreases survival in the mammalian host. PLoS Pathog. 6, e1000932

79 Faghiri, Z. and Skelly, P.J. (2009) The role of tegumental aquaporin from the human parasitic worm, Schistosoma mansoni, in osmoregulation and drug uptake. FASEB J. 23, 2780-2789

80 Faghiri, Z. et al. (2010) The tegument of the human parasitic worm Schistosoma mansoni as an excretory organ: the surface aquaporin SmAQP is a lactate transporter. PLoS ONE 5, e10451

81 Tielens, A.G. et al. (1989) Schistosoma mansoni: rapid turnover of glycogen by adult worms in vivo. Exp. Parasitol. 68, 235-237

82 Bhardwaj, R. and Skelly, P.J. (2009) Purinergic signaling and immune modulation at the schistosome surface? Trends Parasitol. 25, 256-260

83 Bhardwaj, R. and Skelly, P.J. (2011) Characterization of schistosome tegumental alkaline phosphatase (SmAP). PLoS Negl. Trop. Dis. 5, e1011

84 Young, N.D. et al. (2009) Elucidating the transcriptome of Fasciola hepatica - a key to fundamental and biotechnological discoveries for a neglected parasite. Biotechnol. Adv. 28, 222-231

85 Young, N.D. et al. (2010) Unlocking the transcriptomes of two carcinogenic parasites, Clonorchis sinensis and Opisthorchis viverrini. PLoS Negl. Trop. Dis. 4, e719

86 Young, N.D. et al. (2011) A portrait of the transcriptome of the neglected trematode, Fasciola gigantica - biological and biotechnological implications. PLoS Negl. Trop. Dis. 5, e1004

87 Adamidi, C. et al. (2011) De novo assembly and validation of planaria transcriptome by massive parallel sequencing and shotgun proteomics. Genome Res. 21, 1193-1200

88 Tchoubrieva, E. and Kalinna, B. (2010) Advances in mRNA silencing and transgene expression: a gateway to functional genomics in schistosomes. Biotechnol. Genet. Eng. Rev. 26, 261-280

89 Hokke, C.H. et al. (2007) Integrating transcriptome, proteome and glycome analyses of Schistosoma biology. Trends Parasitol. 23, $165-174$

90 Kooij, T.W. et al. (2006) Plasmodium post-genomics: better the bug you know? Nat. Rev. Microbiol. 4, 344-357

91 Grabherr, M.G. et al. (2010) Genome-wide synteny through highly sensitive sequence alignment: Satsuma. Bioinformatics 26, 1145-1151

92 Donthu, R. et al. (2009) SyntenyTracker: a tool for defining homologous synteny blocks using radiation hybrid maps and whole-genome sequence. BMC Res. Notes 2, 148

93 Skovlund, E. and Fenstad, G.U. (2001) Should we always choose a nonparametric test when comparing two apparently nonnormal distributions? J. Clin. Epidemiol. 54, 86-92 